\title{
3D ACOUSTIC LAGRANGIAN VELOCIMETRY
}

\author{
M. Bourgoin \\ L.E.G.I. - U.M.R. 5519 \\ C.N.R.S. / I.N.P.G. / U.J.F. \\ BP53 - 38041 Grenoble Cedex 09 \\ Email: mickael.bourgoin@hmg.inpg.fr
}

\author{
C. Baudet \\ L.E.G.I. - U.M.R. 5519 \\ C.N.R.S. / I.N.P.G. / U.J.F. \\ BP53 - 38041 Grenoble Cedex 09
}

\author{
A. Cartellier \\ L.E.G.I. - U.M.R. 5519 \\ C.N.R.S. / I.N.P.G. / U.J.F. \\ BP53 - 38041 Grenoble Cedex 09
}

\author{
P. Gervais \\ L.E.G.I. - U.M.R. 5519 \\ C.N.R.S. / I.N.P.G. / U.J.F. \\ BP53 - 38041 Grenoble Cedex 09
}

\author{
Y. Gagne \\ L.E.G.I. - U.M.R. 5519 \\ C.N.R.S. / I.N.P.G. / U.J.F. \\ BP53 - 38041 Grenoble Cedex 09
}

\section{ABSTRACT}

We report Lagrangian measurements obtained with an acoustic Doppler velocimetry technique. From the Doppler frequency shift of acoustic waves scattered by tracer particles in a turbulent flow, we are able to measure the full three-component velocity of the particles. As a first application, we have studied velocity statistics of Lagrangian tracers in a turbulent air jet at $R_{\lambda} \sim 320$ and at various distances from the nozzle. The choice of an air jet is motivated by the fact that jets produce a well characterized high level tubulence and open air flows are well suited to simultaneaously achieve classical hot wire Eulerian measurements. Therefore, we are also able to explicitly address the question of the differences between Eulerian and Lagrangian statistics. As Lagrangian tracers we use soap bubbles inflated with Helium which are neutrally buoyant in air and can be assimilated to fluid particles. Velocity statistics are analysed. We show that the Lagrangian autocorrelation decays faster in time than its Eulerian counterpart. Finally we present Lagrangian time velocity increments statistics which, as already reported by previous work, exhibits stronger intermittency than Eulerian velocity increments.

\section{INTRODUCTION}

Experimental and theoretical studies of turbulence has longly been dominated by the Eulerian approach, where a given quantity in the fluid is measured with a probe located at a fixed point in space and is represented as a continuous spatial field. An alternate point of view is given by the Lagrangian approach, where the same quantity is measured along the trajectory of a fluid particle and is represented as a function of time parametrized by the initial position of the considered fluid particle. One reason for the domination of Eulerian approaches in turbulence is probably due to technical difficulties inherent to Lagrangian experiments, which requires the tracking of particles in strongly fluctuating flows. This has remained out of reach of experimentalist until very recently, thanks to technological advances in fast imaging, and ultrasonic technics. Simultaneously theoretical advances of stochastic models for turbulence gave a renewed interest to Lagrangian experiments. Moreover many practical situations are naturally described in the Lagrangian framework. This is particularly the case of dispersion in particle laden flows.

An important advance in Lagrangian measurments have been done in the nineties by Virant and Dracos [1] who developped a 3D-Particle Tracking Velocimetry (PTV) technic. They 
used simultaneously 4 video cameras at a frame rate of $25 \mathrm{fps}$ to access the 3D trajectories of several hundreds of particles at once. Ott and Mann [5] developped a similar technique to study relative dispersion of fluid particles. Because of the low frame rate, particle dynamics could be resolved only for moderate Reynolds numbers, typically $R_{\lambda}<250$, where $R_{\lambda}$ is defined based on the Taylor microscale. The first Lagrangian measurements for single particles statistics in high Reynolds number regimes $\left(R_{\lambda}<1000\right)$ were obtained by LaPorta et al [2], using silicon strip detectors (initially developed for high energy particles detection) at a frame rate up to $70 \mathrm{kHz}$. They observed a strong Lagrangian intermittency characterized by strong acceleration events with non gaussian fluctuations. Recently, Bourgoin et al. [3] have developed a high resolution 3D-PTV facility using ultrafast cameras at a repetition rate of $27 \mathrm{kHz}$, which allows the tracking of several hundred of particles in high Reynolds number regimes $\left(R_{\lambda}<1000\right)$. They studied relative dispersion and Lagrangian structure functions, which also exhibit strong intermittency [18].

All the technics mentioned so far are optical and give the particles position. Particles velocity and acceleration are obtained by differentiating numerically once and twice the position. This is a very noise sensitive procedure which requires an important oversampling in order to get a good signal to noise ratio for the particles velocity and ultrafast optical systems need to be used to study highly turbulent flows. An alternate option is given by ultrasonic technics. Mordant et al [4] used Doppler frequency shift of acoustic waves scattered by tracer particles to obtain Lagrangian velocity in a Von Kármán water flow. The frequency shift is directly proportional to the particle velocity, no differentiation is required to get the instantaneous velocity, and a single differentiation gives the acceleration of the particles. Eventhough the tracers they used were much bigger than the Kolmogorov length of their flow (acoustic scattering impose a minimal tracer size) their results clearly show strong Lagrangian intermittency : the probability density functions of velocity increments are Gaussian for large time separation and strongly non Gaussian for small time separation.

In neither of the previous studies a clean comparison between Lagrangian and Eulerian statistics in turbulent flows were made, either because Eulerian measurements were not available $[2,4]$, or because Reynolds number was too low, so that turbulence can not be considered as fully developed $[1,5,6]$. In the present paper, we describe an experimental setup allowing to perform simultaneous Lagrangian and Eulerian measurements in a turbulent air jet $\left(R_{\lambda} \simeq 320\right)$. Small helium-filled neutral soap bubbles are seeded from a position upstream of the nozzle. The three components of the Lagrangian velocity are obtained by acoustical Doppler effect. First, the detection setup, as well as the tracers are described. Then, we present the signal processing techniques used to detect the passage of bubbles and to extract their velocities from the frequency shift. finally we report some results on the main statistical properties of the velocity signals.

\section{EXPERIMENTAL SETUP}

The measurements have been conducted in a round air jet, emerging from a $2.25 \mathrm{~cm}$ conic nozzle and expanding freely in the room. The flow becomes self-similar at a distance of approximately 40 times the nozzle diameter [7]. Measurement has been done at distances up to 120 diameters.

In all experiments the Reynolds number based on the Taylor microscale is about 320. Jet characteristics are based on hot-wire measurements and classical isotropic relations $[8,9]$.

\section{Particle detection}

Figure 1 presents the principle of one-component ultrasonic velocimetry. It is based on the Doppler frequency shift of an acoustic wave scattered by a moving particle. A transducer emits a continuous ultrasonic wave at a frequency of $110 \mathrm{kHz} \leq v$ $130 \mathrm{kHz}$ with a propagating direction $\vec{n}_{i}$ towards the jet. The wave scattered in a specifc direction $\vec{n}_{d}\left(\theta=\left(\vec{n}_{i} ; \vec{n}_{d}\right)\right.$ is the scatter angle) by particles transported in the flow, is recorded by a receiver. Because of the paricles motion, the scatterd wave is Doppler shifted an its frequency $v^{\prime}$ differs from $v$ so that

$$
\frac{v^{\prime}-v}{v}=\frac{\vec{V} \cdot\left(\vec{n}_{i}-\vec{n}_{d}\right)}{c}=-2 \frac{V_{/ /}}{c} \sin (\theta / 2),
$$

where $c$ is the speed of sound in the experimental conditions. For a given incoming frequency $v$ and a given scatter angle $\theta$, the instantaneaous frequency shift $v^{\prime}-v$ gives a direct measurement of the projection, $V_{/ /}$, of the tracer velocity along $\vec{n}_{i}-\vec{n}_{d}$. Note that this is an algebraic measurement : the sign of $V_{/ /}$is given by the sign of the frequency shift.

The electric signal originating from the receiver is digitized by a HP E1430A card at $65536 \mathrm{~Hz}$ after digital heterodyne demodulation. Series of 1,048,576 samples are recorded. Within each series, several isolated particles are successively detected. Transducers are capacitive electro-acoustical circular piston of Sell-type, with a diameter of $24 \mathrm{~cm}$. They are reciprocal, highly directive and linear. Thanks to the transducers high directivity, tracers can only be detected when they are located in the volume defined by the intersection of the incoming and the detection transducer beams, which will be called in the following the "measurement volume". Its shape is sketched on figure 1. Dimensions are $L_{s} \simeq 50 \mathrm{~cm}$ along the jet axis and $25 \mathrm{~cm}$ across the stream.

One challenge that arises in Lagrangian measurement is to follow the particle over a long enough trajectory in order to describe its dynamics up to scales of order the integral scale of the flow. This means that we seek to record the velocity of a single tracer for a time $T_{S}$ larger than the Lagrangian integral time scale $T_{L}$. A simple order of magnitude calculation gives hints about ways to achieve this experimentally. It was shown numerically [10] that the Eulerian time scale $T_{E}=L_{E} / u^{\prime}$ is related 


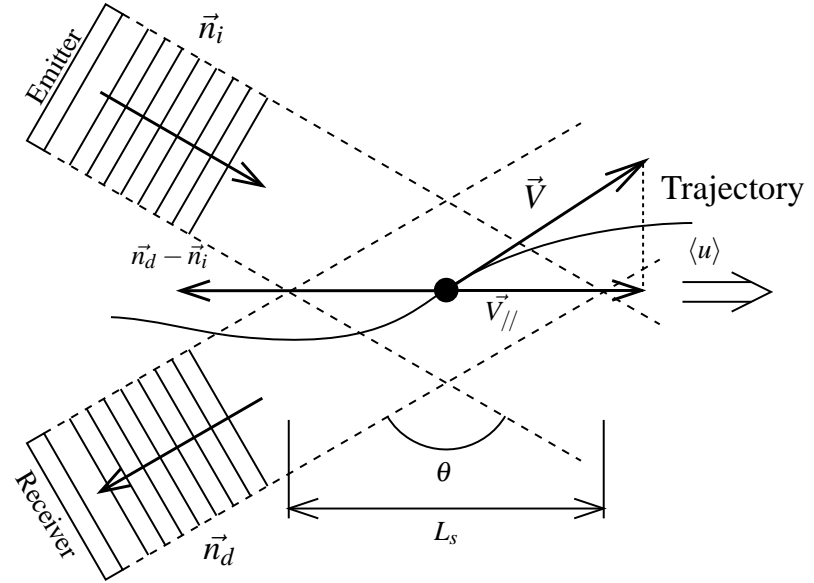

Figure 1. Principle of velocity measurements. Particles can be detected in the intersection of the emitter and receiver acoustic beams (dashed lines). In the configuration we used : $L_{s} \simeq 50 \mathrm{~cm}$.

to the Lagrangian time scale by $T_{L}=0.78 T_{E}$ ( $L_{E}$ is the Eulerian integral length scale and $u^{\prime}$ is the velocity standard deviation). The maximum time-of-flight in the measurement volume is $T_{S} \simeq L_{S} /\langle u\rangle(\langle u\rangle$ is the mean velocity). In the turbulent free jet, the ratio $u^{\prime} /\langle u\rangle$ is about 0.25 , independent of the position and the Reynolds number. Therefore, $T_{S} / T_{L} \simeq\left(L_{S} / L_{E}\right) /(4 \cdot 0.78)$. Maximising $T_{S} / T_{L}$ is then only a matter of maximising $L_{S} / L_{E}$, which is completely independent of the velocity at the nozzle. The Eulerian integral scale $L_{E}$ depends on the distance from the nozzle and on the nozzle diameter. With the chosen value for $L_{s}, L_{s} / L_{E}$ ranges from 8 to 4 , respectively for a measurement volume 60 diameters and 110 diameters downstream from the nozzle. The maximum value for $T_{S} / T_{L}$ is then expected to be between 2.6 and 1.3 .

Small-scale limitation is mainly related to the tracer size. As tracers to study fluid particle dynamics, we use helium inflated soap bubbles (which are density matched with air). At present they can't be made smaller than about $2 \mathrm{~mm}$. This is below the Taylor microscale (from $4.4 \mathrm{~mm}$ to $7.3 \mathrm{~mm}$ ), but still above the Kolmogorov scale $\eta$ (from $0.12 \mathrm{~mm}$ to $0.20 \mathrm{~mm}$ ). Thus, we expect the bubble dynamics to reflect a substantial part of the inertial range, up to the integral scale, but very small scale dynamics might be filtered due to the particles size (previous work suggests that tracers should be smaller than about $5 \eta$ to behave as perfect fluid particles [11]).

\section{Three-component measurement}

It is possible to measure the complete three velocity components by combining several one-component measurements from multiple pairs of acoustic transducers. Four transducers (two emitters and two receivers) are placed at the vertices of a square, tilted so that their axes cross at the same point on the jet axis, in

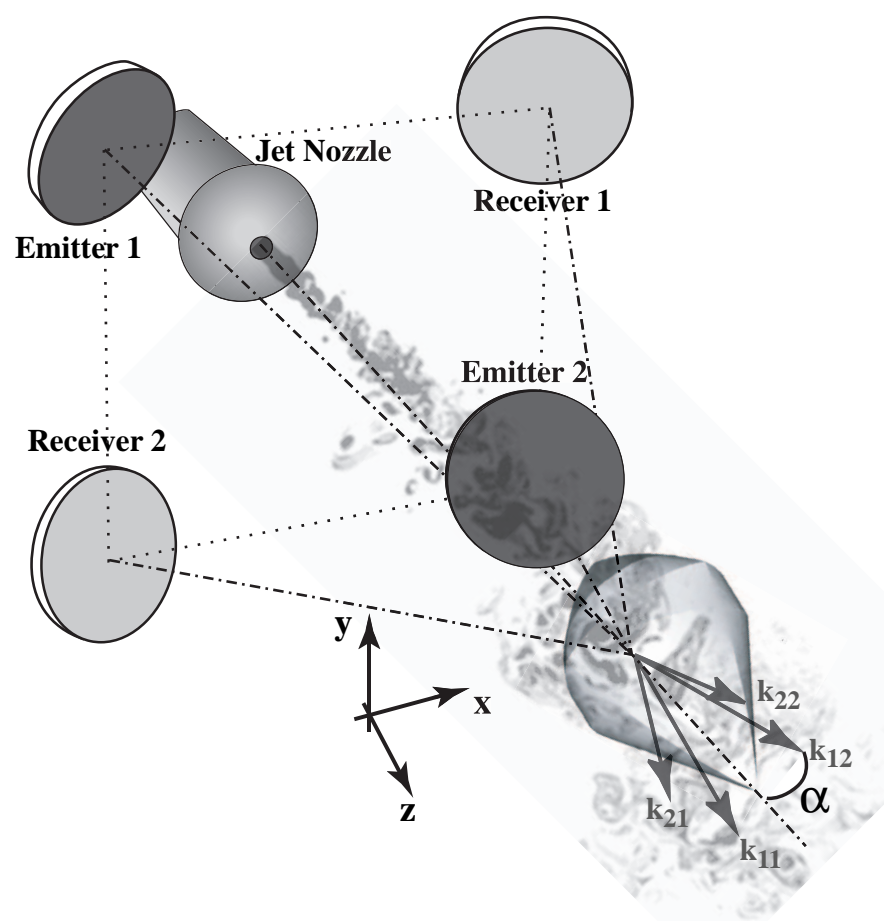

Figure 2. Transducers arrangement for the three-component measurement. $D=24 \mathrm{~cm}, D^{\prime} \simeq 50 \mathrm{~cm}$. Emitters are referred to as $\mathrm{E} 1$ and $\mathrm{E} 2$, receivers as R1 and R2. $\alpha=266 \circ$, yielding a scattering angle $\theta=128^{\circ}$.

a square-based pyramid configuration (figure 2). Distances from transducers to the intersection with the jet axis are all identical, ensuring that wave propagation times are all identical. Transducers are reverse-facing the nozzle, to avoid bubble impacts on their active surfaces and they are sufficiently apart from each other not to pertub the flow.

The first emitter E1 generates a continuous wave at frequency $v_{1}=110 \mathrm{kHz}$, scattered by each bubble, and then recorded in two different directions by the two receivers (resp. $\mathrm{R} 1$ and R2). This gives access to two projections of the tracer velocity, respectively along vectors $\vec{k}_{11}$ and $\vec{k}_{12}$. Similarly, the wave emitted by E2, at frequency $v_{2}=122 \mathrm{kHz}$ (different from $v_{1}$ ) is also scattered and then recorded by the two receivers, giving access to two more projections along vectors $\vec{k}_{21}$ and $\vec{k}_{22}$. Provided the bubble lies in the intersection of the four acoustic beams, its velocity can be obtained through four non-orthogonal projections, which we will denote by $\left(v_{11}, v_{12}, v_{21}, v_{22}\right)$. Components along and perpendicular to the jet axis $(u, v, w)$ can then be computed by an simple matrix transformation.

Using multiple transducers is a major improvement not only because it gives the velocity vector, but also in terms of signal to nois ratio (SNR). The first characteristic that increases the SNR is redundancy. The velocity components $(u, v, w)$, can be simultaneously obtained from the $v_{11}, v_{12}, v_{21}$ and $v_{22}$ by the formulas ( $\alpha$ is defined on figure 2 ): 


$$
\begin{gathered}
u=\frac{v_{11}+v_{12}+v_{21}+v_{22}}{4 \cos \alpha} \\
v=\frac{v_{12}-v_{21}}{2 \sin \alpha} \quad w=\frac{v_{22}-v_{11}}{2 \sin \alpha} .
\end{gathered}
$$

With the assumption that each projection $\left(v_{11}, v_{12}, v_{21}, v_{22}\right)$ is affected by an independant additive random noise of variance $\sigma^{2}$, the variance of the noise for $u$ can be estimated as $\sigma^{2} / 4 \cos ^{2} \alpha \simeq \sigma^{2} / 3.23$, which reveals a real SNR enhancement for the longitudinal velocity component. This is not the case however for the transverse components, $v$ and $w$, for which the variance of the noise is given by $\sigma^{2} / 2 \sin ^{2} \alpha \simeq \sigma^{2} / 0.38$ and remains higher than the noise for the initial projections.

With working frequencies in the $100 \mathrm{kHz}-150 \mathrm{kHz}$ range, acoustic absorption is far from being negligible. Sound paths have to be as short as possible. A second advantage of the pyramidal setup is that transducer separation distance $D^{\prime}$ can be made very short without perturbing the flow, because of the round shape of the jet. For a given scatter angle, acoustic path is then shorter for the three-component measurement than for the singledimensional one.

Despite the transducers high directivity, some acoustic energy still propagates straight from each emitter to each receiver, responsible for a spurious signal at the emitting frequency. As the wave travels across the turbulent flow, it is phase- and amplitudemodulated (scintillation, [12]). A final advantage of the pyramidal setup is that scintillation is greatly reduced. For reasons that will be discussed below, this increases the accessible velocity range and eases the signal processing.

\section{Tracers}

We have first used the acoustic velocimetry technic to study Lagrangian statistics of turbulence. This requires to have Lagrangian tracers which must match the carrier fluid density in order to cancel buoyancy forces. It is a main concern when looking for Lagrangian tracers for air. Whereas solid particles can be used for liquid, particles filled with light gases be used in air. We used helium-inflated soap bubbles. Once the soap film has been made thin enough, the overall bubble density can match the one of air. Helium-inflated bubbles have also the additional benefit of offering a high contrast of acoustic impedance that increases the scattered amplitude.

Due to the evaporation of the liquid film, bubbles life time is around one or two minutes, which is much larger than the time needed to travel across the whole measurement volume (less than $.1 \mathrm{~s})$. Bubbles are produced by a dedicated machine to the desired density, and injected just upstream of the nozzle, so as not to disturb the flow. Statistics for bubble diameters have been obtained with the help of a video camera. It has been found that bubbles diameter has a very monodispersed distribution $(2 \mathrm{~mm} \pm 6 \%$ ). This ensures that limitations due to bubble size will be at a constant scale. Production frequency has to be low enough to ensure that most of the time a single bubble is detected in the measurement volume. Injection rates of about 5 bubbles per second gave good results.

The acoustic technic can also be used to track non Lagrangian particles, in order for instance to study inertial effects. This work is in progress at present. First measurments are planned with heavy bubbles (obtained by thickenning the soap film and/or by filling the bubbles with a heavier gas, such carbon dioxyde).

\section{SIGNAL PROCESSING}

\section{Channels separation}

Figure 3 shows the power spectrum of a typical acoustic signal recorded on one of the receivers over several second (during the period of the record, of order 10 particles have travelled successively in the measurement volume). One observes two sharp high-amplitude spikes exactly at the incoming frequencies. They correspond to direct propagation between emitters and receiver, as well as echoes. Beside each spike, at lower frequencies, stands a broader-band bump which corresponds to Doppler frequencies of the acoustic wave scattered by the particles. The maximum of the band reflects the average velocity of the particles, while its width reflects the velocity fluctuations. All time information is lost in the Fourier space representation of the acoustic signal. The main point of the processing of the acoustic signals will be to obtain simultaneously time and frequency information in order to extract the instantaneous velocity of single particles. This requires first to filter out the spikes (which correspond to direct acoustic waves propagation and don't carry any information on the particles velocity) and to separate the channels for each emitter-receiver pair. Filtering out the spikes removes a small part of the Doppler frequencies because of overlapping. This enforces a limitation on the smallest velocity that can be extracted. Two notch filters are applied to remove the spikes, then a demodulation followed by a low-pass filtering allow the separation of the two scattered signals. We emphasize the fact that demodulation is performed by multiplication with a complex exponential (not a real cosine), yielding an analytic signal. The same operation is applied to the two signals recorded on the two receivers, leading to four different frequency-modulated signals, which will be denoted hereafter by $s\left(s_{i} \ldots s_{l}\right)$. 


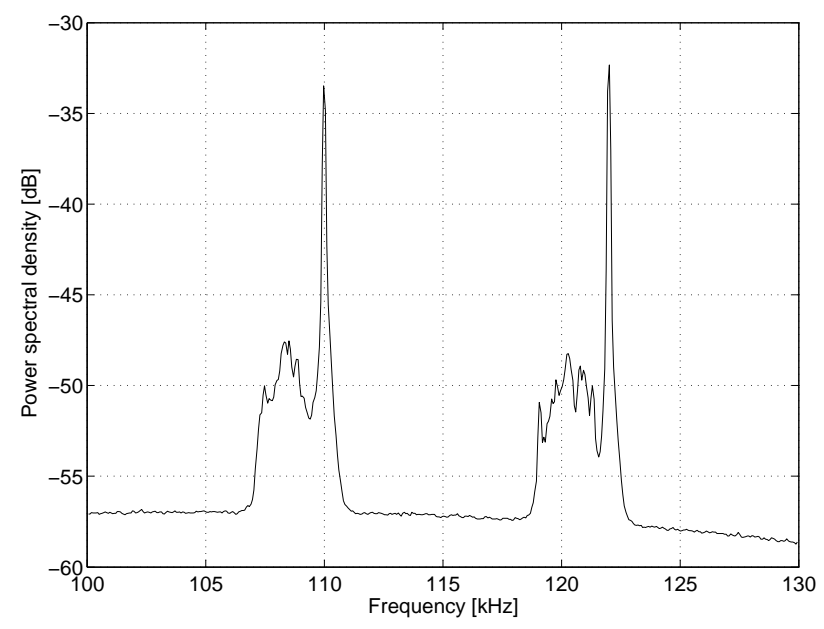

Figure 3. Power spectral density of the signal recorder by one of the receivers. The two spectral lines are the emitter direct frequencies $(110 \mathrm{kHz}$ and $122 \mathrm{kHz}$ ), and correspond to acoustic waves propafating straight between the emitters and the receiver.

\section{Time detection of tracers}

The acoustic signal is recorded as successive aquisitions which are typically 20 seconds long. During each aquisition, about 100 bubbles travel in the measurement volume. Only the portions of signal corresponding to the presence of a particle in the measurement volume are analyzed for extraction of Lagrangian velocity. Figure 4 shows the real part of a typical $s$ signal (only a fraction of order 0.8 second is represented). We observe that the signal amplitude (computed as the complex magnitude) is very close to the enveloppe of the real part, confirming the analytical property of the signal. The presence of a bubble in the measurement volume corresponds to high amplitude events easily identifiable on the figure. When no bubble is present, amplitude is not strictly zero. This remaining amplitude is mostly due to sound scattering by the vorticity field (see [13]) and will be considered as noise here.

Automatic detection of bubbles is achieved by a thresholding operation. Time intervals are determined by the following algorithm :

- Low-pass filtering of the signal amplitude $(\sim 4000 \mathrm{~Hz})$;

- Computation of the median of the former, as an estimation of noise level ;

- Selection of time intervals where filtered amplitude is above twice the noise level ;

- Rejection of intervals shorter than 10 samples ;

- Merging of intervals separated by no more than $5.3 \mathrm{~ms}$ (350 samples) ;

- Rejection of intervals shorter than $30 \mathrm{~ms}$ (2000 samples).

This operation is applied independently on each of the $s$ signals.

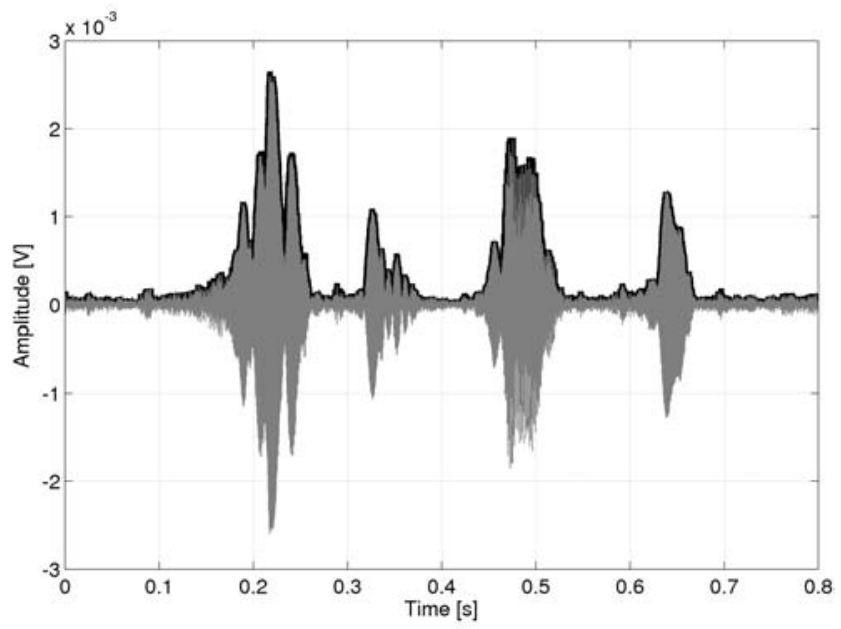

Figure 4. Real part (grey line) and amplitude (black line) of separated acoustic signal $(s)$.

For the velocity vector to be computed, the bubble should be detected at least on three signals simultaneously. Another selection procedure is thus applied to keep only intervals that have a common part on the four channels.

\section{Signal to noise ratio maximisation}

As explained before, the signal of interest is the wave scattered by the bubble. Noises include

i) wave propagating straight between emitter and receiver and echoes;

ii) scattering by the vorticity field;

iii) overall electronic noise;

iv) turbulent acoustical noise.

In practice, iii) and iv) are of little concern. Acoustical amplitude can be made sufficiently large so that noise iii) is negligible. Noise iv) has only frequencies below $10 \mathrm{kHz}$, which is far from the band of study $(100 \mathrm{kHz}-150 \mathrm{kHz})$.

Once noise iii) has been overcome, increasing emitting power does not improve SNR, because signal and remaining noises i) ii) are all proportional to the incoming sound amplitude. No way to reduce noise i) has been found so far.

Reducing noise ii) can be achieved by adjustement of the diffusion angles and of the working frequency. When the frequency increases, the scattered amplitude decreases (see [13]). Similarly, for a $90^{\circ}$ diffusion angle, the scattered amplitude vanishes. Experiments have shown that bubble scattering cross section does not drop in the accessible frequency range, so that increasing the frequency increases the SNR. Above $130 \mathrm{kHz}$, sound absorption raises significantly, making noise iii) more and more important. As a consequence, time intervals detected shorten (because the beginning and the end of segments are hidden by noise). 
The final setup $(\theta=128$ degrees, frequencies between $110 \mathrm{kHz}$ and $130 \mathrm{kHz}$ ) is the result of a compromise between the following constraints :

- Longest measurement volume (largest $\theta$ )

- Lowest noise on the transverse components (smallest $\theta$ )

- Lowest vorticity scattering amplitude (highest frequency, $\theta$ near $\pi / 2$ )

- Lowest sound absorption (smallest $\theta$, lowest frequency)

One can appreciate a typical SNR on figure 4.

\section{Extracting velocity from acoustic signal}

In order to extract the instantaneous velocity of the individual tracers, we have to combine the spectral and the time represetantions of the acoustic signal. Indeed, the spectral representation loses all time information and can only give time-averaged information such as the mean velocity of the bubbles (which corresponds to the peak of the Doppler shift) and the time representation is well suited to detect in time individual tracers travelling in the measurement volume but it doesn't give any information on their velocity. The determination of the instantaneous velocity of the tracers relies on an estimation of the instantaneous frequency of the acoustic signal. Cohen class energetic estimators are classical tools for this purpose. We chose the Choï-Williams distribution, for its moment-preserving property ( [14]). If $x$ is a complex-valued signal, its Choï-williams distribution $C_{x}$ is defined by:

$$
\begin{aligned}
\forall t, f \quad C_{x}(t, f) \hat{=} & \int_{-\infty}^{\infty} \int_{-\infty}^{\infty} \frac{\delta}{|\tau|} \mathrm{e}^{-2 \delta^{2}(s-t)^{2} / \tau^{2}} \\
& x\left(s+\frac{\tau}{2}\right) x^{*}\left(s-\frac{\tau}{2}\right) \mathrm{e}^{-2 \mathrm{i} \pi f \tau} \mathrm{d} s \mathrm{~d} \tau
\end{aligned}
$$

$\delta$ is a parameter. We used $\delta=1$.

A sample result is shown on figure 5. Frequency is along the vertical axis, time along the horizontal. Grayscale levels quantifies the energy level. Two crooked lines are visible, they correspond to two distinct bubbles. The lines where the signal energy is concentrated reflect the time evolution of the instantaneous Doppler frequency shift. The time-frequency transformation (4) gives a 2D representation from which the instantaneous frequency shift $v^{\prime}(t)$ is extracted as the frequency average weighted by the energy distribution $C_{s}$ :

$$
\forall t, \quad v^{\prime}(t)=\frac{\int_{-\infty}^{\infty} f C_{x}(t, f) \mathrm{d} f}{\int_{-\infty}^{\infty} C_{x}(t, f) \mathrm{d} f}
$$

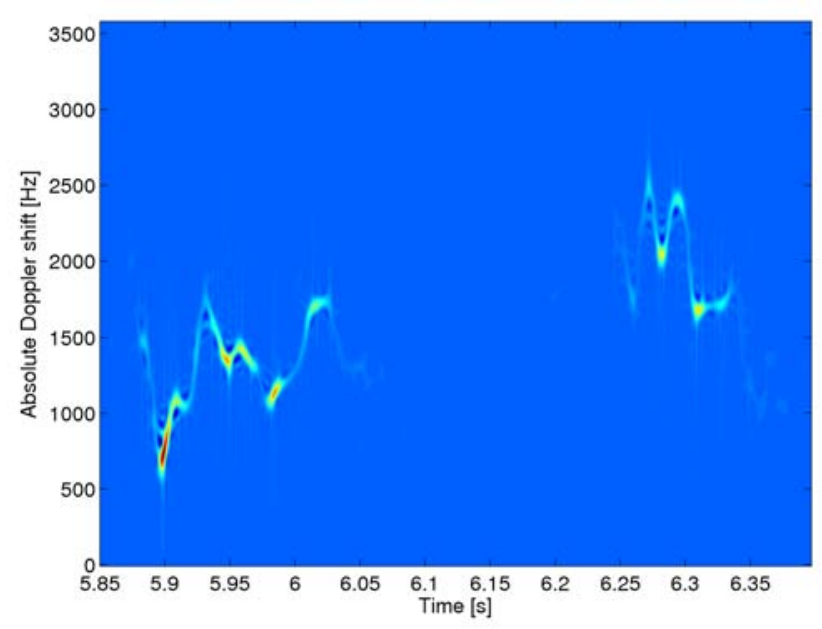

Figure 5. Time-frequency representation. Two bubble signals are visible.

Then, equation (1) directly gives the corresponding velocity component.

This velocity extraction is applied to each $s$ signal, on the selected intervals. Coordinate transformation yields lots of small (2000 to 8000 points) velocity signals which will be called "velocity segments" in the following. No time continuity exists between velocity segments, they all correspond to different bubbles. Such a procedure leads to a large set of independent realizations of Lagrangian velocities. The $j$-th point (time) of the $i$-th segment (realization) will be denoted by $\vec{v}_{i}(j)$.

\section{RESULTS \\ Data set}

As already discussed, in order to resolve not only the small scale dynamics but also the large scale dynamics of the particles we need the measurement volume dimensions to be comparable to the integral scale of the flow. Therefore, we carried the experiments in an air jet with a small nozzle $(2.25 \mathrm{~cm}$ in diameter) compared to the transducers diameter. As a consequence, only moderate Reynolds number (up to $R_{\lambda}=320$ ) were achievable. Series of recordings were made at four distances $(D)$ from the nozzle. Every measurement corresponds to the same Reynolds number, as it is constant along the jet, but to different integral length scales [7-9]. The measurement volume was centered on the jet axis, to preserve cylindrical symmetry as much as possible.

Table 1 lists the main parameters of the different measurements. The number of velocity segments exceeds 1000 for all measurements, ensuring good statistical convergence. Measurement volume length is several times larger than the Eulerian integral length scale $\left(L_{S} / L_{E}\right)$, and the ratio of the maximal timeof-flight to the expected Lagrangian integral time scale $\left(T_{S} / T_{L}\right)$ 


\begin{tabular}{ccccccc}
$D$ & $\mathrm{~N}$ & $L_{E}[\mathrm{~cm}]$ & $T_{L}[\mathrm{~ms}]$ & $\frac{L_{s}}{L_{E}}$ & $\frac{\left\langle T_{s}\right\rangle}{T_{L}}$ & $\frac{T_{s}}{T_{L}}$ \\
\hline 58 & 3552 & 6.2 & 35 & 8.1 & 1.48 & 2.6 \\
80 & 9584 & 8.8 & 60 & 5.7 & 1.22 & 1.8 \\
93 & 7444 & 10.3 & 80 & 5.0 & 1.20 & 1.6 \\
111 & 1358 & 12.3 & 120 & 4.2 & 1.02 & 1.3
\end{tabular}

Table 1. Experimental parameters at different distances $D$ from the nozzle and the center of the measurement volume. $D$ is measured in multiples of the nozzle diameter. $N$ is the number of velocity segments.

is everywhere above one. This estimation is in good agreement with the experimental ratio $\left\langle T_{s}\right\rangle / T_{L}$, which involves the mean time-of-flight and the measured Lagrangian integral time scale. Values of $\left\langle T_{s}\right\rangle / T_{L}$ decreases with increasing nozzle distance, as expected.

\section{Probability density function}

The normalized velocity probability densty functions (PDF) for the longitudinal velocity component measured at different distance from the nozzle are represented on figure 6. No significant change in shape can be seen between the four curves, indicating that the variation of $L_{s} / L_{E}$ does not break self-similarity. The same remark is true for transverse components (figure 7). All curves are Gaussian, but small departures exist. For the longitudinal component (figure 6), PDF edges are largely sub-Gaussian due to limitations of the velocity extraction algorithm and has no physical meaning. For transverse components (figure 7), edges are over-Gaussian because of noise introduced by the velocity extraction algorithm. Previous studies by [4] have also shown that the Lagrangian velocity PDF in a Von-Kármán flow has a Gaussian shape.

Figure 8 shows the comparison of the Lagrangian PDF $(P(u)$ ) with the corresponding Eulerian one (the hot-wire was located near the center of the Lagrangian measurement zone). A reasonable agreement is found. A slightly higher mean velocity is found in the Eulerian case (5\% higher), and the standard deviation is higher for the Lagrangian velocity. These effects results from the inhomogeneity of the flow inside the acoustic measurement volume, which tend to under estimate the Lagrangian mean velocity on the axis and over estimate its fluctuations but isn't visible on the Eulerian measurement which is carried at a fixed point.

Figure 9 shows isocontours of the joint PDF $P(u, v)$ of longitudinal $u$ and transverse $v$ Lagrangian velocity. A slightly elliptical shape is visible, indicating that no large-scale isotropy exists (horizontal and vertical coordinates are identical). Standard deviation of the longitudinal component is higher than the corre-

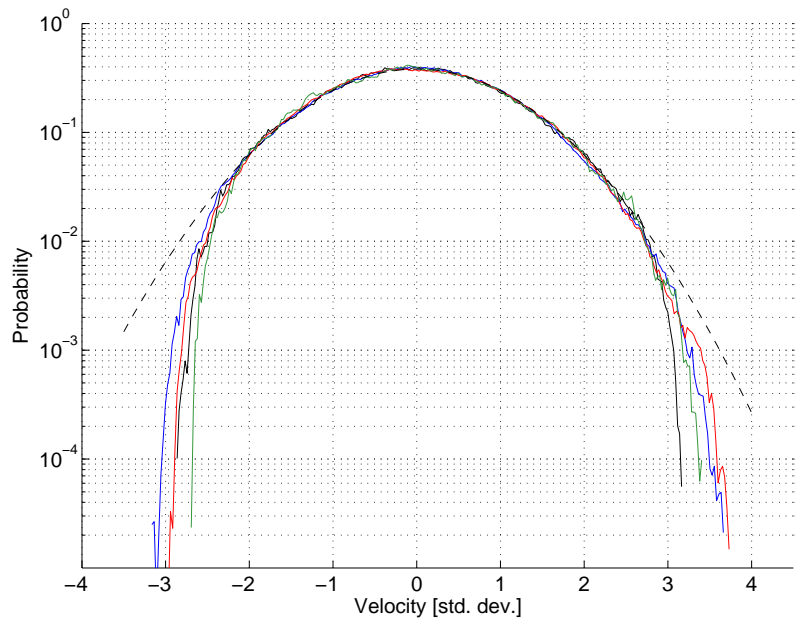

Figure 6. Longitudinal velocity PDF with zero mean and unity variance. Corresponding Gaussian curve is plotted in dashed line.

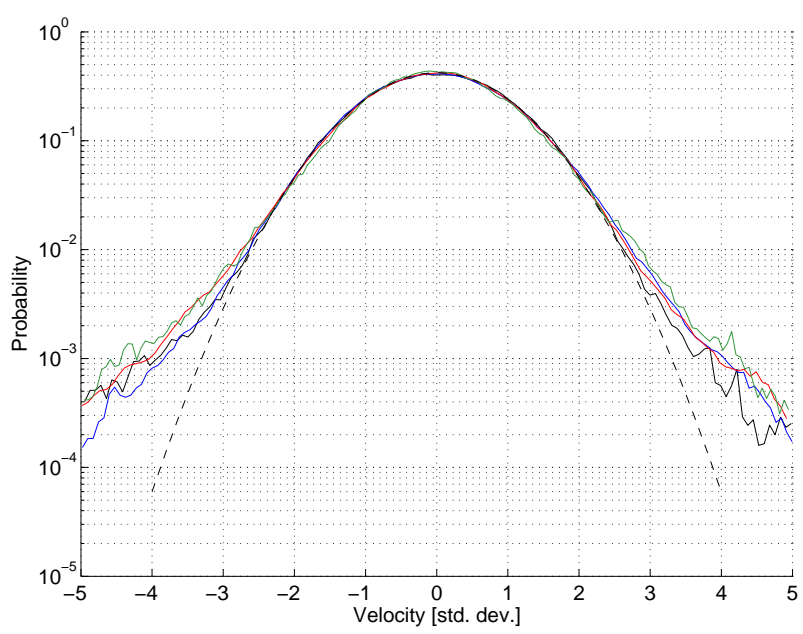

Figure 7. Transverse velocity PDF with zero mean and unity variance. Corresponding Gaussian curve is plotted in dashed line.

sponding one for the transverse component, by a factor ranging from 1.1 to 1.25 , depending on the position along the jet (resp. farthest and nearest from the nozzle). A similar behaviour exists for Eulerian velocity components (see [7]) This non-constant ratio can also be explained by the variation of the ratio between the lateral size of the measurement volume and the local transverse integral length scale $L_{E}$.

\section{Statistical estimation of correlation}

Lagrangian statistics tend to be biased towards the lower velocities, because slowest particles spend more time in the measurement volume (this is the same phenomenon that biases laser 


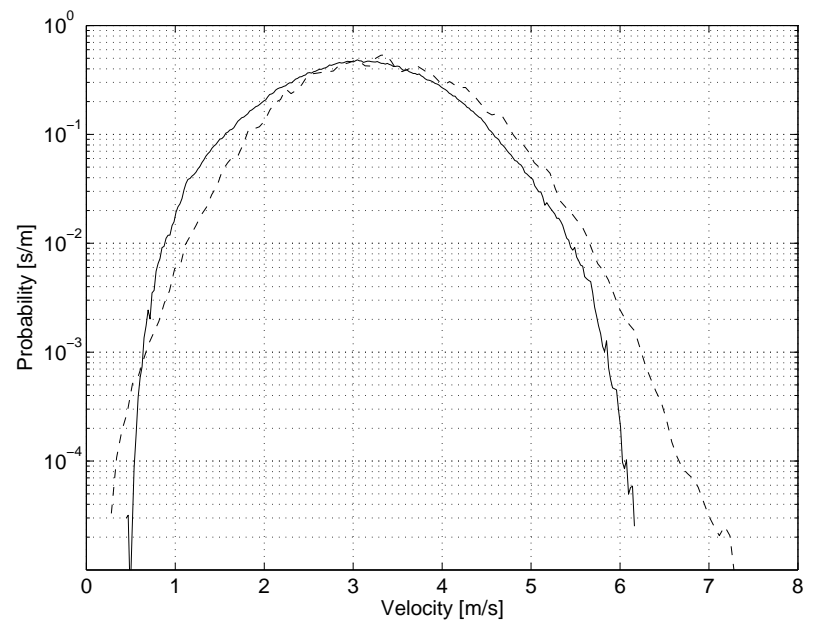

Figure 8. Lagrangian (solid line) and Eulerian (dashed line) velocity PDF (80 diameters from the nozzle).

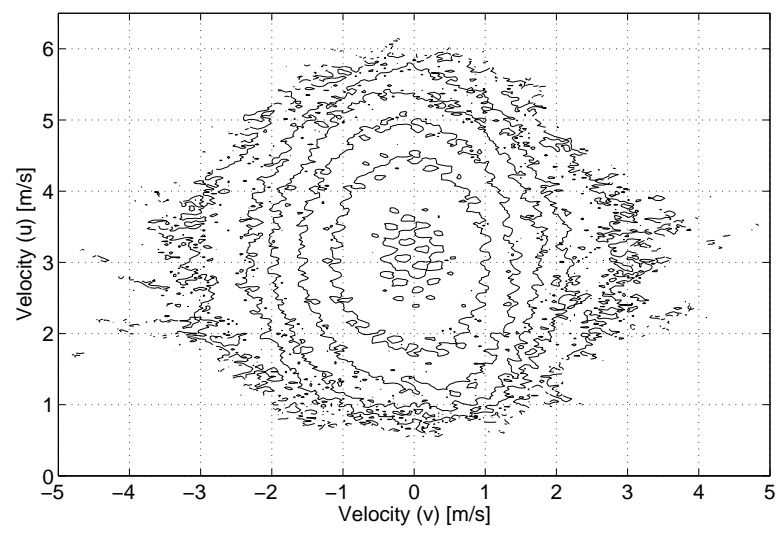

Figure 9. Isocontours of joint Lagrangian velocity PDF. Contour values, from the center outward, are $10^{-3.5}, 10^{-4}, 10^{-4.5}, 10^{-5}, 10^{-5.5}$, $10^{-6.5}$.

Doppler velocimetry measurements). This bias is a particular issue when correlation functions are to be estimated, because values for the longest time lags can only be computed with the longest segments, which corresponds to the slowest particles. In order to compensate fot this bias, the velocity correlation function is estimated with the following formula :

$$
\begin{aligned}
& \forall l \forall k, \quad R_{l l}(k) \widehat{=} \frac{1}{(\vec{\sigma})_{l}^{2} \sum_{i / L i>k} L_{i}-k} . \\
& \sum_{i / L_{i}>k} \sum_{j=1}^{L_{i}-k}\left[\left(\vec{v}_{i}(j)\right)_{l}-\langle\vec{v}\rangle_{l}\right]\left[\left(\vec{v}_{i}(j+k)\right)_{l}-\langle\vec{v}\rangle_{l}\right] .(6)
\end{aligned}
$$

where $L_{i}$ is the length of segment $i, \vec{v}^{>}(k)_{l}$ and $\vec{\sigma}^{>}(k)_{l}$ are the mean velocity and standard deviation for the l-th component of the velocity, estimated from segments longer than $k$ only :

$$
\begin{gathered}
\forall k=1 . . \max _{i} L_{i}, \quad \vec{v}^{>}(k) \widehat{=} \frac{\sum_{i / L_{i}>k} \sum_{j=1}^{L_{i}} \vec{v}_{i}(j)}{\sum_{i / L_{i}>k} L_{i}} \\
\forall k, l, \quad\left(\vec{\sigma}^{>}(k)\right)_{l} \widehat{=} \sqrt{\frac{\sum_{i / L_{i}>k} \sum_{j=1}^{L_{i}}\left(\vec{v}_{i}(j)\right)_{l}^{2}}{\sum_{i / L_{i}>k} L_{i}}-\left(\vec{v}^{>}(k)\right)_{l}^{2}}
\end{gathered}
$$

\section{Correlations}

Figure 10 shows the autocorrelation function of the Lagrangian velocity components and the Eulerian longitudinal velocity for a measurement performed at 80 diameters from the nozzle. The two curves for Lagrangian transverse components are almost identical, in accordance with the cylindrical symmetry of the flow. The longitudinal component exhibits a slightly longer time scale.

We denote in the following the longitudinal and transverse Lagrangian integral time scales by $T_{L}^{l}$ and $T_{L}^{t}$ respectively. These values are computed by fitting an exponential curve on the autocorrelation. Corresponding values for Eulerian components are denoted by $T_{E}^{l}$ and $T_{E}^{t}$. Only $T_{E}^{l}$ can be readily obtained from measurements: $T_{E}^{l} \widehat{=} L_{E}^{l} / \sigma_{E}^{l}$, where $L_{E}^{l}$ is the Eulerian integral length scale (obtained from the autocorrelation and Taylor hypothesis), and $\sigma_{E}^{l}$ is the standard Eulerian longitudinal standard deviation. As no measurement of transverse Eulerian velocity has been performed, $T_{E}^{t}$ is estimated from the longitudinal value, assuming that $L_{E}^{l} / L_{E}^{t} \simeq 2.3$ and $\sigma_{E}^{l} / \sigma_{E}^{t} \simeq 1.2$ (as found in [7]).

All these values are listed in table 2 for the different positions of the measurements. We note that the transverse integral time scales are smaller than the longitudinal. In the Eulerian case, the ratio $T_{E}^{l} / T_{E}^{t}$ is constant as a consequence of the previous hypotheses. On the contrary, we observe that ratio $T_{L}^{l} / T_{L}^{t}$ tends to increase with the distance $D$ from the nozzle. Several reasons may be responsible for that. On the one hand, the jet self-similarity can be broken. [7] have shown that self-similarity can be violated for distances as large as 100 nozzle diameters, depending on the quantity considered, in which case actual measurements of Eulerian time scales would lead to similar results. On the other hand the velocity profile varies linearly with the distance to the nozzle, while the measurement volume size is constant, so that the flow homogeneity in the measurement volume depends on the position in the jet. As $T_{L}^{l} / T_{L}^{t}$ increases when $D$ increases, this indicates that large-scale isotropy either does not exist whatever the distance, or is recovered very slowly. Lagrangian times $T_{L}$ can be considered as a rough measure of eddy 
life-time, whereas $T_{E}$ is related to the eddy turnover time. These results show that whatever the component considered, both times are very close, the turnover time being slightly longer. Obtained ratios are compatible with the predicted value of $1 / 0.78 \simeq 1.28$.

A simple phenomenological analysis leads to $T_{L} \simeq T_{E}$ [15]. A larger Eulerian time scale can be explained by sweeping effects. The advection of the internal scales by the energycontaining scale leads to broadening of the Eulerian autocorrelation in comparison with the Lagrangian one [16]. This explains the increase of the Eulerian integral scale.

\begin{tabular}{c|ccc|ccc|cc}
$D$ & $T_{L}^{l}$ & $T_{L}^{t}$ & $\frac{T_{L}^{l}}{T_{L}^{t}}$ & $T_{E}^{l}$ & $T_{E}^{t}$ & $\frac{T_{E}^{l}}{T_{E}^{t}}$ & $\frac{T_{E}^{l}}{T_{L}^{l}}$ & $\frac{T_{E}^{t}}{T_{L}^{t}}$ \\
\hline 58 & 35 & 26 & 1.35 & 48 & 25 & 1.92 & 1.37 & 0.98 \\
80 & 62 & 49 & 1.27 & 98 & 51 & 1.92 & 1.58 & 1.04 \\
93 & 79 & 59 & 1.34 & 129 & 69 & 1.92 & 1.63 & 1.16 \\
111 & 117 & 75 & 1.56 & 205 & 98 & 1.92 & 1.75 & 1.30
\end{tabular}

Table 2. Eulerian and Lagrangian time scales in milliseconds. $T_{L}^{l}, T_{L}^{t}$ and $T_{E}^{l}$ measured. $T_{E}^{t}$ computed from $T_{E}^{l}$ (see text).

Cross correlations of transverse Lagrangian components $(v, w)$ are shown on figures 11. All curves are very close to zero (noise floor is about 0.05), showing that no correlation exist between the lagrangian velocity components, despite the high inhomogeneity of the flow in the measurement volume. This supports one-dimensional modeling of fluid particle large-scale motion. Large-scale decorrelation is also an a posteriori validation of our measurement setup: if measurements of the four velocity projections $(\mathrm{i}, \mathrm{j}, \mathrm{k}, \mathrm{l})$ were made on a non-regular pyramid, a residual cross-correlation would exist.

\section{Velocity increments and intermittency}

We define the velocity increments signal for a given time lag $\tau$ as

$$
\delta \vec{v}(\tau, t)=\vec{v}(t+\tau)-\vec{v}(t) .
$$

Figure 12 represents the longitudinal Lagrangian velocity increments PDF for different time lags $\tau$. We note that large scale increments PDF is gaussian and that it deviates from the gaussian as $\tau$ decreases, developing tails close to exponential. This behavior reflects the strong Lagrangian intermittency already reported in previous works $[11,17,18]$. Particularly, the small scale increments reflects particles acceleration and the exponential tails are

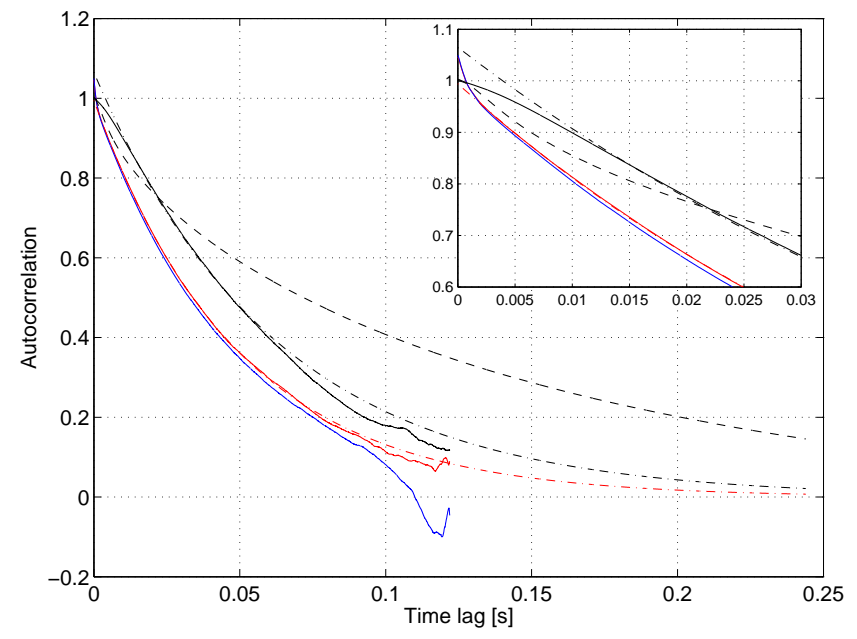

Figure 10. Lagrangian velocity autocorrelation (solid line) for longitudinal and transverse components. Eulerian velocity autocorrelation (dashed). An exponential fit has been superimposed to Lagrangian correlations (dotdashed).

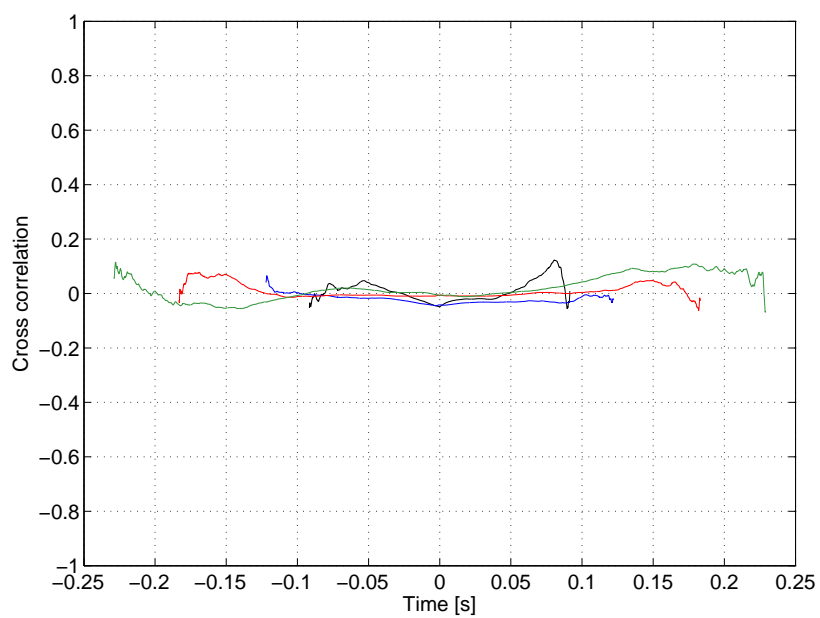

Figure 11. Cross correlation of Lagrangian velocities $(v, w)$.

characteristic of very high acceleration events which occur with a probability higher than gaussian.

Figure 13 represents the kurtosis (fourth moment of the PDF normalized by its variance) of the velocity increments as a function of the time lag, for Lagrangian and Eulerian longitudinal velocity. For large increments, both the Eulerian and Lagrangian kurtosis tend to a value equal to 3 , what corresponds to a gaussian PDF as expected for large scales. As we look at smaller scales increments, the flatness increases, what reflects the intermittency as the PDF develops non gaussian tails. Both Eulerian and Lagrangian statistics are intermittent, but the Lagrangian intermittency appears to be significantly stronger. It is also worth 


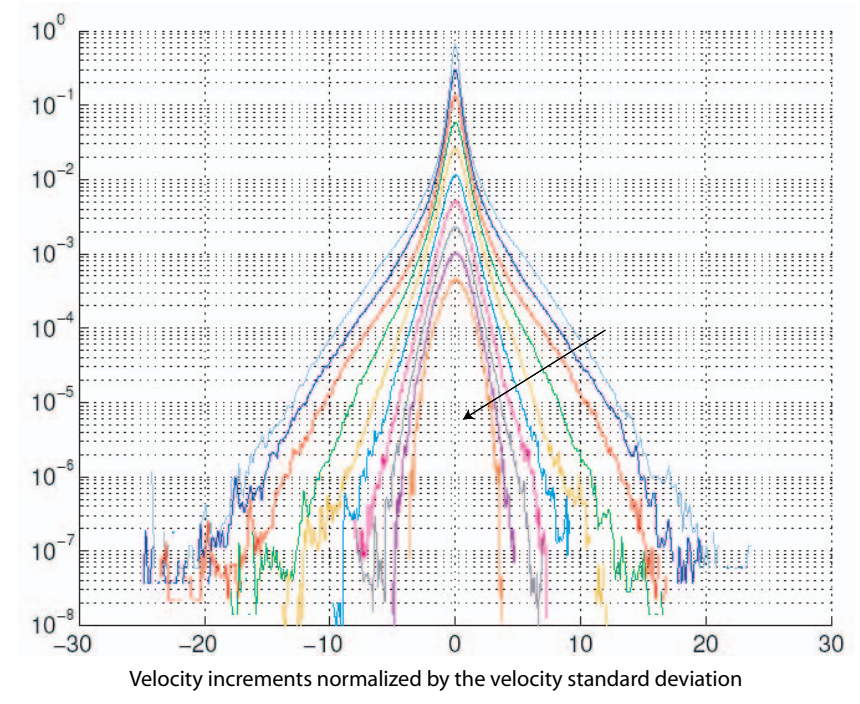

Figure 12. Longitudinal Lagrangian velocity increments probability density functions normalized to variance equal to 1 . The time lag $\tau$ increases as indicated by the arrow from $0.3 \mathrm{~ms}$ to $90 \mathrm{~ms}\left(\tau_{\eta}=1.8 \mathrm{~ms}\right.$, $T_{L} \simeq 60 \mathrm{~ms}$ ).
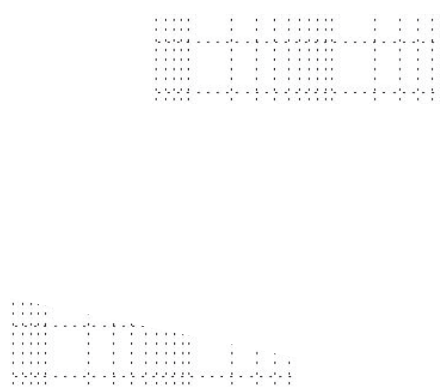

Figure 13. Kurtosis of the longitudinal Lagrangian $(\circ)$ and Eulerian $(\times)$ velocity increments. (Measurements at different positions from the nozzle have been superposed).

noticing that even if the finite size of our tracers might filter part of the small scales Lagrangian dynamics, the measured intermittency is still very robust.

\section{CONCLUSION}

Lagrangian measurements in a free turbulent air jet were performed using acoustical Doppler effect. This method is adapted to collecting large data sets without tremendous memory requirement, contrary to visualisation method. A single tracer at a time can be detected, with the time- and space- dynamics of the measurements comprising a large part of the inertial scales, comparable to previously-obtained results ( [4]). Simultaneous Eulerian measurements were performed.

We show that the Eulerian integral time is larger than the Lagrangian one, what can be a consequence of the Eulerian statistics sensitivity to sweeping effects, which instead do not affect Lagrangian statistics. This result holds for distances in the jet ranging from 60 nozzle diameters up to 110 nozzle diameters.

The analyze of velocity increments exhibits strong Lagrangian intermittency, with highly non gaussian fluctuations at small scales, reflecting the existence of strong acceleration events along the particles trajectory.

The acoustic technique is now being adapted to study two phase flows ladden with inertial particles. The first experiments aim to explore Stokes number dependance of individual particles dynamics, with a particular focus on the effect of particles finite size and of the particle to fluid densities ratio.

\section{ACKNOWLEDGMENT}

This work is fully supported by the French Ministère de la Recherche and by the Université Joseph Fourier (PPF plateforme expérimentale de spectroscopie acoustique multi-échelles). We thank Michel Lebey for kindly lending us their bubble machine. We wish to acknowledge fruitful discussions with Jean-Francois Pinton and Laurent Chevillard. We also thank Jean-Paul BarbierNeyret and Joseph Virone for their valuable technical help.

\section{REFERENCES}

[1] Virant, M., and Dracos, T., 1997. "3D PTV and its application on lagrangian motion". Measurement science and technology, 8, pp. 1539-1552.

[2] LaPorta, A., Voth, G. A., Crawford, A. M., Alexander, J., and Bodenschatz, E., 2002. "Fluid particle accelerations in fuly developped turbulence". Nature, 409, February, p. 1017.

[3] Bourgoin, M., Ouellette, N. T., Xu, H., Berg, J., and Bodenschatz, E., 2006. "The role of pair dispersion in turbulent flow". Science, 311, February, p. 835.

[4] Mordant, N., Metz, P., Michel, O., and Pinton, J.-F., 2001. "Measurement of lagrangian velocity in fully developed turbulence". Physical Review Letters, 87(21), p. 214501.

[5] Ott, S., and Mann, J., 2000. "An experimental investigation of the relative diffusion of particle pairs in threedimensional turbulent flow". Journal of Fluid Mechanics, 422, pp. 207-223.

[6] Sato, Y., and Yamamoto, K., 1987. "Lagrangian mea- 
surement of fluid-particle motion in an isotropic turbulent field". Journal of Fluid Mechanics, 175, pp. 183-199.

[7] Wygnanski, I., and Fiedler, H., 1969. "Some measurements in the self-preserving jet". Journal of Fluid Mechanics, 38(3), pp. 577-612.

[8] Pope, S. B., 2000. Turbulent flows. Cambridge University Press.

[9] Tennekes, H., and Lumley, J. L., 1992. A first course in turbulence. MIT press.

[10] Yeung, P. K., 2002. "Lagrangian investigations of turbulence”. Annual Review of Fluid Mechanics, 34, pp. 115142.

[11] Voth, G. A., la Porta, A., Crawford, A. M., Alexander, J., and Bodenschatz, E., 2002. "Measurement of particle accelerations in fully developed turbulence". Journal of Fluid Mechanics, 469, pp. 121-160.

[12] Monin, A. S., and Yaglom, A. M., 1975. Statistical fluid mechanics. MIT press.

[13] Poulain, C., Mazellier, N., Gervais, P., Gagne, Y., and Baudet, C., 2004. "Spectral vorticity and lagrangian velocity measurements in turbulent jets". Flow, Turbulence and Combustion, 72, pp. 245-271.

[14] Flandrin, P., 1993. Temps-fréquence. Hermès.

[15] Corrsin, S., 1963. "Estimates of the relations between Eulerian and Lagrangian scales in large Reynolds number turbulence". Journal of the Atmospheric Sciences, 20(2), pp. 115-119.

[16] Kraichnan, R. H., 1964. "Relation between lagrangian and eulerian correlation times of a turbulent velocity field". Physics of Fluids, 7(1), pp. 142-143.

[17] Mordant, N., Delour, J., Léveque, E., Michel, O., Arnéodo, A., and Pinton, J.-F., 2003. "Lagrangian velocity fluctuations in fully developed turbulence: scaling, intermittency and dynamics". Journal of Statistical Physics, 113, pp. 701-717.

[18] Xu, H., Bourgoin, M., Ouellette, N. T., and Bodenschatz, E., 2006. "High order lagrangian velocity statistics in turbulence". Physical Review Letters, 96, January, p. 024503. 